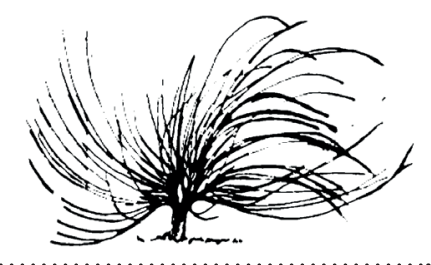

\title{
Integración del pensamiento freireano al quehacer universitario: hacia una transformación de la propia praxis
}

\author{
Cinthya Olivares Garital \\ Universidad Nacional \\ Costa Rica \\ coliga@una.ac.cr
}

«El proceso liberador no es tan sólo un crecimiento profesional: es una transformación al mismo tiempo social y de uno mismo, un momento en el que "aprender" $y$ "cambiar la sociedad"

caminan juntos»

(Freire y Shor, 2014, p. 85).

\begin{abstract}
Resumen
A partir de las ideas de Paulo Freire y de otros autores mencionados en el desarrollo de este ensayo, se realiza un análisis crítico de las falencias propias del personal docente universitario y del sistema educativo en general. Se exploran cuatro acciones transformadoras inspiradas en las ideas freireanas de dialogicidad, pedagogía de la pregunta, el binomio dialéctico reflexión-acción y la alfabetización crítica. El objetivo del presente escrito es propiciar una autoconciencia en el cuerpo docente, con el fin de liberar su praxis de los vicios tradicionalistas y propiciar la transformación del estudiantado, como producto de esa liberación.
\end{abstract}

Recibido: 20 de mayo de 2019. Aprobado: 28 de octubre de 2019.

http://dx.doi.org/10.15359/rep.15-1.3

1 Magíster en Segundas Lenguas y Culturas con énfasis en Inglés como Lengua Extranjera para Alumnado Adulto y magíster en Gestión Educativa con énfasis en Liderazgo de la Universidad Nacional de Costa Rica. Doctorando en Educación con énfasis en Educación Emocional de la Universidad Internacional Iberoamericana de México. 
Palabras clave: pensamiento freireano, alfabetización crítica, reflexión-acción, dialogicidad, pedagogía de la pregunta.

\begin{abstract}
Based on Pablo Freire's thinking and other experts' mentioned in this essay, the author analyzes critically university professors' weaknesses and the educational system's in general. Four transforming actions inspired by Freire's ideas about dialogicity, pedagogy of the question, the reflection-action dialectical binomial, and critical literacy are explored. This essay aims to foster self-awareness in university professors for the purpose of freeing their praxis from traditional vices and boosting the transformation of the students as a result of that liberation.
\end{abstract}

Keywords: Freire's thinking, critical literacy, reflection-action, dialogicity, pedagogy of the question.

\title{
Introducción
}

$\mathrm{L}$ a educación formal ha sido un arma poderosa de transformación social. A través de ella una sociedad es capaz de expandir su conocimiento científico, cultural y tecnológico. Lastimosamente, es también utilizada como instrumento de manipulación ideológica de las masas. Es decir, los controles despóticos sobre lo que se debe aprender y cómo debe hacerse en los centros de estudio son dictados por ideologías absolutistas. Ante esta paradoja de la educación, surge el pensamiento freireano influenciado por las ideas filosóficas de Hegel, Husserl, Gramsci y Marx. Sus creencias fundamentan el aprendizaje centrado en el estudiantado y su realidad social, así como la pedagogía crítica y transformadora (Masi, 2008).

Para Hegel (1991), la educación debe tener su punto de partida, el cual, ciertamente, es la humanización mediante la formación ética. Humanizar conlleva al cuerpo docente y estudiantil mismo a comprender su posición dentro de la sociedad a la cual pertenece. De Hursserl, filósofo representante de la fenomenología transcendental, se rescata la noción de la consciencia intencional. Su obra evoca el retorno al uso de la razón como instrumento esencial para lograr la superación. Esta conciencia intencional devela un proceso de concienciación y utilización 
de la criticidad, en el que el sujeto transciende "la esfera espontánea de la aprehensión de la realidad para llegar a una esfera crítica en la cual, la realidad se da como objeto cognoscible y el hombre asume una posición epistemológica" (Sáiz, 2003, p. 122).

La filosofía de Antonio Gramsci, quien junto con Freire es reconocido por sus aportes a la educación radical, establece una crítica al sentido común. Desde su línea de pensamiento, el sentido común no es más que un conformismo ante la realidad, lo que representa un gran desafío. Gramsci (1972) se enfrenta al reto de reemplazar el sentido común por una filosofía de la praxis. Freire incorpora parte de este desafío en su definición de praxis conformada por el binomio dialéctico e indivisible reflexión-acción. Por último, Marx es quien apuesta por el rol activo del sujeto cognoscente en el ciclo gnoseológico definido por Freire (Santos, 2008). Es decir, en la relación trabajo-producción, la persona construye el conocimiento al actuar y someter el pensamiento a la acción.

Pablo Freire es quien, a través de su discurso y sus ideas, ha provocado una transformación revolucionaria, mas no reaccionaria, del acto educativo. Ha impregnado de ideales y despertado el coraje y la osadía del cuerpo docente, en su lucha por un cambio social desde las aulas. Su aporte político e intelectual a la educación, si bien surgió en la década de los 60, aún hace eco resonante en las mentalidades abatidas por la ideología opresora que prevalece. La ideología neoliberal, mercantil y capitalista ha enajenado el potencial de la mente humana a lo largo de los años en nuestra sociedad costarricense. Liberar la mente es el primer paso de la emancipación propia de la esclavitud política y social, según Gramsci (1972), y es Freire quien da fundamento pedagógico para que tanto el personal docente como el estudiantado inicien el camino hacia esa liberación.

\section{Al rescate de la educación liberadora: praxis según Freire}

Si se partiera del supuesto de que, "es necesario que quien sabe, sepa sobretodo que nadie sabe todo y nadie ignora todo" (Freire, 1982, p. 27), se podría llegar a un convencimiento de la incapacidad de conocer todo y de la existencia de la reciprocidad en el acto educativo. En otras palabras, el cuerpo docente y el estudiantado se necesitan mutuamente para transformarse, para liberarse a través de su propia praxis. Desde la perspectiva freireana, la praxis conlleva la infalible vinculación de 
la relación acción-reflexión-acción. El sujeto interpreta su realidad, su vida y, a partir de esta interpretación, ejerce su práctica más conscientemente, provocando una acción transformadora a posteriori (Streck, Redín y Zitkoski, 2010). Esta apropiación de la vida mediante el análisis crítico le permite a cada uno la modificación de su realidad.

Praxis es, por lo tanto, el resultado de una actuación consciente y responsable que rompe con la alienación y la domesticación, para dar paso a la transformación de uno mismo y del propio entorno. La perspectiva freireana sobre la praxis rompe con el paradigma educativo de nuestra sociedad actual. ¿En qué medida la teoría es internalizada no solo para ser luego arrojada en una prueba, sino más bien interpretada, aprehendida y puesta en acción para transformar? Esa es una interrogante digna de un análisis exhaustivo.

Ahora bien, ¿en qué medida el colectivo estudiantil comprende que la educación no es un acto del uno para el otro sino una práctica hecha en comunión (Streck, Redín y Zitkoski, 2010)? Basada en esta última inquietud, la perspectiva del conocimiento y del ciclo de construcción de este debe variarse. El saber no es un producto finalizado y en su construcción participan tanto el discente como el docente. Con este enunciado se pone fin a la contradicción educador-educando y se convierte en una correspondencia indisoluble, en la cual tanto el estudiante se convierte en educador como el docente en educando, en el proceso gnoseológico citado por Freire.

La praxis evocada por Freire reconoce la grandeza de la palabra de cada uno de los actores del ciclo gnoseológico. La interpretación de la realidad reviste al sujeto de propiedad para ejercer el poder de la palabra y, por lo tanto, de ejercitar el derecho de ser escuchado y de provocar una liberación. A medida que se produzca una liberación de la consciencia, se produce una social (Streck, Redín y Zitkoski, 2010). La provocación de esta es el tapiz que debe imperar en cada acto educativo, en el cual se pretenda dignificar a ambos sujetos (docente-discente), elevando su esencia a una transformación propia y de su entorno.

\section{Transformación de la praxis universitaria}

La responsabilidad que yace en las manos del personal docente es de proporciones significativas. Si tan solo los educadores pudiéramos percatarnos del poder otorgado a través de la profesión misma de formar o de-formar a los discentes, otra consciencia surgiría al ejercer 
nuestra tarea educativa. Freire destaca que la educación pensada en virtud de la humanidad comprende "una rigurosidad metódica, la investigación, el respeto a los saberes del educando, la conciencia del inacabamiento, la curiosidad, el saber escuchar y la disponibilidad para el diálogo" (Streck, Redín y Zitkoski, p. 187, 2010). Además, la óptica de Kant (1983), quien establece que la formación hace a los seres humanos, fundamenta el papel protagónico del cuerpo docente en el proceso educativo enaltecido por Freire: uno de liberación y transformación.

Pero, ¿cómo ocasionamos una educación liberadora, crítica y transformadora basada en los apuntes de Freire, si el panorama educativo universitario parece estar anquilosado y ser restrictivo, incuestionable, vertical, carente de creatividad y criticismo? El anhelo del corazón y el pensamiento de Paulo Freire es aún el mismo anhelo de muchos educadores en la actualidad. Entonces, sí hay esperanza. Primeramente, ante un panorama educativo castrado de curiosidad, imaginación y que hipertrofia los sentidos (Streck, Redín y Zitkoski, 2010), no queda más que el pronunciamiento de un personal docente osado, valiente, decidido y, sobre todo, comprometido con la profesión y la sociedad.

Por tanto, es así como el sistema educativo que describe Freire no excluye al nuestro. Desafortunadamente, se percibe en nuestras aulas universitarias la ausencia de criticidad, creatividad, intuición y entusiasmo en el estudiantado, así como muchos de estos elementos en el cuerpo docente. Aun cuando el modelo pedagógico distintivo de la Universidad Nacional procura la escucha y conversación reflexiva entre el personal docente y el colectivo estudiantil, el uso de metodologías y prácticas pedagógicas innovadoras, el desarrollo de un comportamiento tanto crítico como reflexivo y la generación de una actitud emprendedora en los futuros profesionales (Universidad Nacional, 2012), las falencias de educadores(as) y educandos son evidentes.

El querer transformar y ser transformado, a través de la educación, demanda un acto de valor y humildad. Se requiere fortaleza para realizar una radiografía de nuestra propia praxis y aceptar que, aunque sean muchos los años y los títulos, aún hay mucho por mejorar en esta área. En este sentido, es menester tener en cuenta que la lectura del legado de Freire le permite al lector llegar a un convencimiento de la necesidad de un cambio y mejoramiento continuo urgentes. ¿Cómo iniciar la ruta hacia la praxis descrita por Freire en aras de contribuir a una 
autosuperación y transformación del estudiantado y el cuerpo docente universitario? El punto de partida es la realización de un autoanálisis y luego de un seguimiento cuidadoso de cuatro acciones transformadoras que la sabiduría freireana nos ha heredado: la dialogicidad en acción, la pedagogía de la pregunta, el binomio reflexión-acción y la alfabetización crítica.

\section{Dialogicidad en acción}

Es la educación un pilar fundamental que moldea y transforma al sujeto. De esta transformación participan activamente tanto el docente como el discente. Según Freire (1996), "quien enseña aprende al enseñar y quien aprende, enseña al aprender" (p. 25). Para perpetuar la existencia armoniosa de la relación recíproca enseñar-aprender, el diálogo adquiere una relevancia esencial.

Bajo esta premisa, ¿estamos los integrantes del cuerpo docente universitario dispuestos a dialogar con el estudiantado? La respuesta más expedita es una negación aparente por parte del profesorado. ¿Por qué no estamos dispuestos a dialogar? Posiblemente, exponernos a interrogantes del estudiantado desnuda nuestra esencia y revela quiénes realmente somos, nos hace ver débiles y nos toma por sorpresa. Es decir, el personal docente, al ser cuestionado, se llena de miedos e incertidumbre.

Freire y Shor (2014) dan respuesta a las interrogantes anteriores. El profesorado no está "preparado para evaluar sus propias habilidades como líderes de debates, escuchan pocos conferenciantes estimuladores y críticos, y no reciben capacitación para preparar la voz en ningún arte dramático de más confianza en su propia creatividad verbal" (p. 75). Es decir, los docentes se escudan detrás de los métodos tradicionalistas, como la clase expositiva, para no enfrentar la incertidumbre y el temor que le generaría una clase dialógica. Por otro lado, el cuerpo estudiantil parece haber sido silenciado, producto de la pasividad y rigidez con la que el sistema lo ha formado. Está lleno de temor a ser expuesto al ridículo cuando formula preguntas "erradas". La inactividad y represión del estudiantado ocurren al instaurar una pedagogía transgresora del pensamiento libre y creativo.

Freire establece un enfoque digno de atención para quienes pretendemos iniciar un cambio hacia una pedagogía liberadora. Todo inicia con el saber escuchar. Esta simple acción resta autoritarismo al discurso 
vertical del cuerpo docente y transforma el acto del diálogo de "quien habla para los educandos en la horizontalidad a quien habla con los educandos" (Streck, Redín y Zitkoski, 2010, p. 461). El estudiantado merece ser escuchado y, desde su realidad, puede brindar una luz que no es más tenue que ninguna otra. Este empoderamiento del cuerpo estudiantil para adentrarse en un diálogo se genera a partir del convencimiento del profesorado sobre esta necesidad.

Con el afán de integrar este aspecto del discurso freiriano al quehacer universitario como propuesta de transformación de la propia praxis, es necesario realizar tres pasos sencillos extraídos de la lectura del discurso de Freire. Primeramente, aprender y enseñar a escuchar. Luego, partir de la realidad del estudiantado para contextualizar el contenido y facilitar el aporte de este durante el diálogo (problematización) y, por último, utilizar las lecturas y contextualizarlas. Esta práctica es de aplicación general en los cursos del currículo de cualquier carrera, especialmente en aquellos en los cuales el peso teórico es de consideración.

El dialogar, una vez horizontalizado el discurso, más que una disposición conlleva una acción. Existen acciones facilitadoras de un diálogo más efectivo. Dentro y fuera del aula, se pueden establecer mesas dialógicas disciplinarias e interdisciplinarias. Las primeras pueden ser realizadas por el cuerpo estudiantil y docente de una misma carrera. Esta práctica, en la que todos con una misma voz puedan exponer sus puntos de vista sobre su realidad como estudiante o profesor, contribuiría al desarrollo personal y, por ende, profesional del personal docente y del colectivo estudiantil en conjunto. Pueden realizarse mesas dialógicas disciplinarias entre el estudiantado, entre el personal docente y, mixtas, de estudiantes con docentes. Todas las tres generarían grandes aportes al crecimiento y al progreso académico tanto del estudiantado como del cuerpo docente involucrado.

Además, la creación de mesas dialógicas interdisciplinarias produciría un mayor impacto en la comprensión del papel fundamental de la universidad y su aporte a la sociedad, tomando en cuenta los diferentes saberes. Basados en esta práctica, se asumiría el reto de Freire de "construir nuevos saberes a partir de la situación dialógica que provoca la interacción y la división de mundos diferentes, pero que comparten el sueño y la esperanza de construir juntos nuestro ser más" (Streck, Redín y Zitkoski, 2010, p. 153). 


\section{La puesta en práctica de una pedagogía de la pregunta}

Desde la óptica de Freire (2013), aún hoy prevalece la pedagogía de la respuesta sobre la pedagogía de la pregunta. El estudiantado encuentra a su disposición datos excesivos de los cuales no se plantea ningún cuestionamiento y ni siquiera muestra curiosidad por preguntar. Es decir, hay muchas respuestas para todo, pero pocas preguntas construidas críticamente. Pareciera ser que la educación bancaria o tradicionalista ha paralizado el cuestionamiento del educando, a través de la rigidez de sus prácticas. Al imperar una educación transmisiva, el colectivo estudiantil solo se retrata como un recipiente de la información dispuesto a ser llenado por el conocimiento transmitido por el personal docente. En esta medida, "los educadores y los educandos se archivan, se anulan... no hay creatividad, no hay transformación, no hay saber" (Freire, 1987, p. 58).

Igualmente, Gadamer (1994) concibe la construcción de preguntas como el arte de pensar para la construcción del conocimiento. Ahora bien, en el plano educativo, preguntar es un recurso pedagógico de relevancia que no ha recibido el valor ni la atención merecidos. Para Freire, "todas las preguntas merecen respuesta, y nosotros quizá, nunca tengamos la respuesta, si no una de las respuestas. Debemos evitar el dogmatismo y la falta de respeto" (Freire y Shor, 2014, p. 277). Esto es quizá parte del empoderamiento que debe suceder en el cuerpo estudiantil y la actitud con la que el conjunto docente debe abrazar el acto educativo. Enseñar a preguntar equivale a enseñar a pensar. Es decir, pensar conlleva una orientación del discente hacia la reflexión (interpretación y comprensión de la realidad) anterior al planteamiento de la pregunta; una vez formulados los cuestionamientos y dado el intercambio de estos, se produce la construcción del conocimiento y una posible transformación del sujeto y su realidad.

Lastimosamente, no solo el sistema educativo ha estancado la creatividad y la curiosidad para preguntar. Nuestra cultura propia ha retraído nuestro actuar, nuestro asombro y nuestra motivación para preguntar. Desde pequeños se nos ha negado el derecho a preguntar y esto se refleja en los salones de clases. Nuestros cursos, en muchos casos, están llenos de jóvenes introvertidos, poco arriesgados y temerosos. El cambio del panorama de favorecer una pedagogía de la pregunta es vital, cuando se procura una transformación de la praxis universitaria. 
El inicio de este cambio radica en las prácticas que el estudiantado realiza tanto dentro como fuera del aula. Una de las acciones necesarias para lograr una transformación del colectivo estudiantil es la implementación de "la clase invertida" o "the flipped classroom". Este método centrado en el estudiantado consiste en convertir la instrucción directa en indirecta, empoderando al cuerpo estudiantil a investigar y cuestionarse fuera del salón de clases, a través de las TIC, como primer paso, y luego realizando trabajos y actividades de clase, como la resolución de conflictos, en las cuales el personal docente se convierte solamente en guía. Al final de este ciclo, el profesorado consolida el aprendizaje a través de un resumen en forma de cierre.

Este tipo de aprendizaje es un paso hacia la ruptura de la educación controlada únicamente por el cuerpo docente. La clase invertida genera reflexión, pensamiento crítico, comprensión de contenidos, aprendizaje colaborativo entre pares y aumenta el compromiso (Mattis, 2014). En un programa de curso universitario, de cualquier índole, el aula invertida se puede integrar. Al llegar al nivel de educación superior, el estudiantado ha adquirido la madurez para responsabilizarse por el primer paso, es decir, la investigación de un tema. Además, actualmente, la mayor parte del cuerpo estudiantil universitario ha desarrollado competencias sobre el uso de los recursos tecnológicos, de una manera efectiva y responsable.

Otra acción posible para fomentar la construcción de preguntas críticas es la implementación del método socrático en el análisis de las lecturas de clase. Este es utilizado para profundizar la comprensión de un contenido, a partir de preguntas generadas sobre dicho tema. Un integrante del cuerpo estudiantil selecciona un tema, los demás participantes lo interrogan hasta convencerlo de que sabe menos de lo que pensaba sobre el contenido en un principio. En consecuencia, se entabla un diálogo socrático. Las preguntas formuladas son mezcladas con un poco de ironía hasta llevar a la persona interrogada a cuestionarse a sí misma de lo que inicialmente creía o hasta a contradecir su primera respuesta. El cometido de este diálogo socrático es generar pensamiento cada vez más complejo. Los pasos de este método se detallan a continuación:

En el primero, un interlocutor A (un docente) da una tesis o afirmación que la contraparte considere incierta, lo cual lleva a un análisis y su refutación. En segundo lugar, la contraparte 
manifiesta su opinión y sus premisas, es decir, aquello que sustenta su punto de vista. A continuación, el primer interlocutor argumenta y la contraparte reconoce que los razonamientos que ha ofrecido son contrarios a la primera afirmación. Finalmente, la contraparte puede demostrar con sus proposiciones que la tesis del interlocutor A es falsa y que por lo tanto su negación es verdadera (Zetina-Esquivel y Piñón-Rodríguez, 2017, p. 84).

Este método favorece el desarrollo de habilidades como la crítica, la imaginación, la reflexión, la creatividad y la empatía (Zetina-Esquivel y Piñón-Rodríguez, 2017), además de ser una opción de construcción de preguntas.

\section{Reflexión- acción}

El sujeto cognoscente en la filosofía freireana (2014) es reconocido por características como la acción, la reflexión crítica, la curiosidad, el cuestionamiento exigente, la inquietud y la duda. Para este pedagogo todos conocemos algo e ignoramos algo; por lo tanto, el conocimiento no es lineal, sino más bien cíclico y dinámico, siempre en construcción, al igual que los individuos que lo cimientan. En el proceso de construcción del saber, el profesorado desempeña un papel determinante. Como parte del binomio reflexión-acción, la praxis cumple su razón de ser, al asumir que la práctica y la teoría son indisolubles y que permanecen en constante diálogo (Streck, Redín y Zitkoski, 2010). El estudiantado, debido a su formación tradicionalista en el aula, asume la construcción del conocimiento como un proceso estático y carente del roce con la realidad.

Desde esta perspectiva, es conveniente que la teoría sea ligada a la práctica y viceversa, sin concebirlas como dimensiones separadas ni alejadas una de la otra. En el quehacer universitario, el profesorado es el agente que puede devolver este sentido a la praxis. Este puede guiar al colectivo estudiantil a rehacer su propio proceso de aprender a conocer. Únicamente esto se podrá implementar cuando el cuerpo docente asuma su posición en el acto educativo y su deseo por guiar al estudiantado hacia una transformación de su pensamiento; tal como lo señala Freire (1993), “el proceso de enseñar, que implica el de educar y viceversa, involucra pasión de conocer que nos inserta en una búsqueda placentera, aunque nada fácil" (p. 11). 
Específicamente, una propuesta clave para la integración de la reflexión-acción en nuestro quehacer universitario es la realización de la técnica ateneos didácticos. Ellos se conciben como pequeños espacios de reflexión y acción, a través de la ejecución de reuniones en las que se dan a conocer diferentes puntos de vista y conflictos. El papel de las personas participantes es debatir posibles soluciones al conflicto o respuestas a las cuestiones planteadas (Alen, 2013). Esta técnica produce la reflexión entre pares y una socialización de los saberes. Se cumple, de esta forma, el principio del respeto a la palabra del otro; principio por el que tanto abogó Freire en toda la extensión de su discurso.

Por ejemplo, como parte de la carrera Enseñanza del Inglés de la Universidad Nacional de Costa Rica (igualmente puede ser incluido en cualquier otro curso de práctica docente), el estudiante-profesor cumple con la impartición de 50 clases en una institución educativa y la asistencia a una sesión semanal de seminario en la universidad. Cada semana, después de la clase que el estudiante practicante desarrolle y como parte de las actividades del seminario, se puede plantear la técnica ateneo didáctico. Los estudiantes se reúnen en subgrupos, dialogan sobre los conflictos, incertidumbres e inquietudes que enfrentaron durante su día de práctica. Cada integrante del subgrupo participa en este momento de reflexión, al plantear una resolución al conflicto o una respuesta a la inquietud expuesta. Las diferentes resoluciones al conflicto o respuestas a las inquietudes se registran en una memoria de clase. Posteriormente, el personal docente se da a la tarea de revisar y complementar o ampliar, con su experiencia, dichas memorias.

Como se ha argumentado, esta acción transformadora garantiza la lectura crítica de los problemas y la ejecución inmediata de una solución para modificar la realidad. Los estudiantes practicantes están expuestos al devenir del día tras día, lo cual les brinda la oportunidad de contrastar la teoría con lo vivido en el preciso momento que algún suceso se gesta. A través de este roce con la realidad, resultaría propicio iniciar el ciclo freireano teoría-palabra-acción, por medio del ateneo didáctico.

Otra acción transformadora descrita por Freire y digna de mención es el cierre de clase a través de un resumen o síntesis. Después de un análisis introspectivo, se podría concluir que como docentes nuestra atención ha estado centrada en el inicio y el desarrollo de las clases únicamente, o sea, el cierre no ha recibido el tratamiento adecuado. 
Muchas podrían ser las excusas, pero dos de ellas son el "trajín del día" y la distribución del tiempo. Entonces, ¿es un cierre tan importante como un inicio de clase? Desde la perspectiva de Freire, sí. Para él y Shor (2014), se debe estimular el final a través de un resumen, el cual puede "convertirse, entonces, en un acto de reflexión entre iguales, en lugar de ser un sello protocolar del profesor sobre la hora de trabajo" (p. 245). Es decir, el cierre puede estar a cargo tanto del docente como del estudiantado, de manera que se convierta el momento en un espacio de construcción y síntesis del conocimiento adquirido.

En el mismo sentido, una técnica de reflexión que puede ser incorporada a los fines de clase es el planteamiento de Cerletti (2003), al establecer tres preguntas esenciales que el profesorado debe formular a sus estudiantes: "Y tú... ¿qué ves?, ¿qué piensas?, ¿qué harías?" El autor plantea que la liberación del colectivo estudiantil sucedería gracias a que "las respuestas, entonces, dejarán de ser un secreto que atesora el maestro para transformarse en una conquista, de cada alumno, sobre los saberes, sobre el mundo y sobre sí mismo" (p. 147). Estas tres interrogantes pueden ser utilizadas para crear un cierre de clase efectivo. Significa, entonces, que cada vez que se realice un final o conclusión de una clase o actividad, se brindará un espacio para que el estudiantado participe y pueda crear el resumen o clausura, a través de la utilización de las tres cuestiones anteriores. El objetivo de esta acción transformadora es procurar el empoderamiento del cuerpo estudiantil, partiendo del valor y la grandeza de su palabra.

\section{Alfabetización crítica}

La alfabetización crítica es la capacidad de leer más allá de las palabras, "es hacer de la reflexión crítica de la sociedad una actividad fundamental, es evitar el vuelo sobre las palabras... que impida aprender cómo se relaciona el aprendizaje con la realidad" (Freire y Shor, 2014, p. 141). Para lograr alfabetizar críticamente al estudiantado, debemos cuestionarnos si tenemos la respuesta a las siguientes inquietudes, antes de la ejecución de una actividad de lectura: ¿En qué medida conocemos al ciudadano que la sociedad nos demanda formar? ¿Cómo y para qué necesitan mis estudiantes la información expuesta? ¿Qué valores extraerá el estudiantado de la lectura que les permita efectuar una liberación de su conciencia? ¿Contribuye esta lectura a la formación de una conciencia 
más crítica, reflexiva y propositiva? ¿Concibo a mis estudiantes promotores de un cambio o acción social, después de leer el texto?

En efecto, esta serie de preguntas y quizá otras más que el profesorado se pueda plantear encauzan el deseo del cuerpo docente por generar criticidad, a través de las lecturas. En este mismo sentido, se recomienda analizar el impacto del contenido de cada texto incluido en el plan de clase. Una vez que el colectivo estudiantil toma el tiempo para leer, se le dirige a replantearse el contenido, es decir, si se describe un hecho, bien podría preguntarse "¿Por qué sucedió de esta manera y no de la otra?" o "¿Cuál fue la razón del comportamiento de (personaje)?" "¿Existe otra forma de hacerlo?”. Luego, se le solicita al estudiantado realizar una investigación pequeña acerca de las cuestiones formuladas.

\section{Propuesta para la integración del pensamiento freireano al queha- cer universitario}

Pablo Freire destaca insistentemente varios aspectos estratégicos que el profesorado puede implementar para obtener resultados óptimos en pro del colectivo estudiantil. Algunos de estos aspectos fueron señalados como acciones transformadoras del pensamiento freireano a lo largo de este ensayo: dialogicidad, pedagogía de la pregunta, reflexión-acción, y alfabetización crítica. Precisamente, he planteado aquí esas cuatro acciones principales que, desde mi experiencia docente, atribuyo como urgentes y vitales en la labor universitaria. Reconozco 

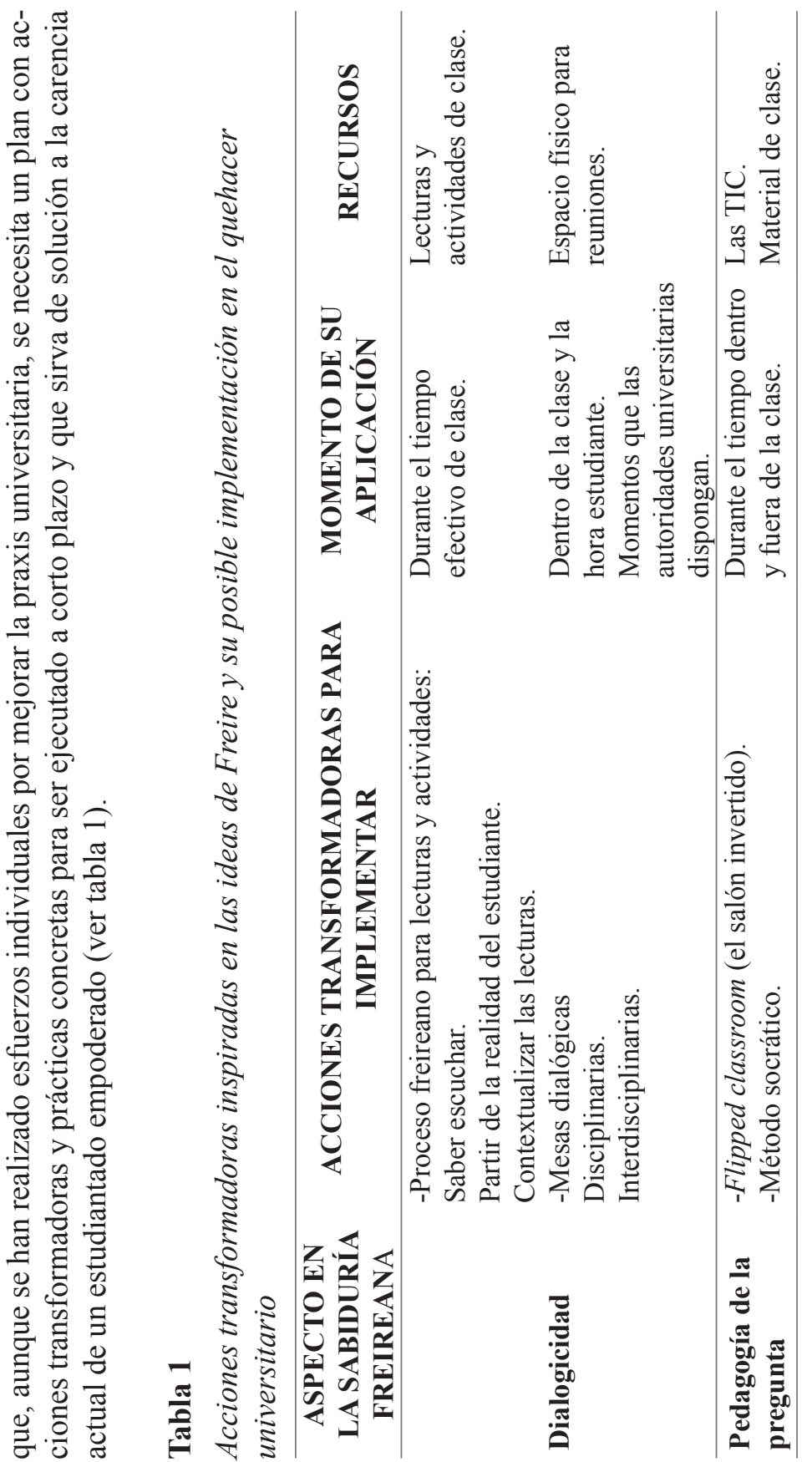
¿qué harías? (Cerletti, 2003).

-Solicitud a las autoridades para ejercer un espacio

de reflexión pedagógico-institucional para docentes

y alumnos.

-Realización de lecturas y cuestionamientos críticos. Durante el tiempo dentro consultas planteadas.

Nota: Elaboración propia, 2018.

Es necesario recalcar que este plan de acciones transformadoras no tiene validez por sí solo. Para su implementación requiere dos voluntades: la del profesorado y la del estudiantado. Asimismo, estas acciones, como parte de una pedagogía crítica y creativa, cobran un gran sentido en la medida en que el cuerpo docente se compromete con su papel liberador y lucha por ello al igual que el colectivo estudiantil.

En concreto, la ejecución de estas acciones liberadoras implica esfuerzo y sacrificio para consolidar el acto educativo como un proceso en el que tanto educador como educando se construyan a sí mismos, simultáneamente. Este proceso se convierte para Freire (1992) y muchos de los casos, "[en] un parto...un parto doloroso. El hombre que nace de él es un hombre nuevo" (p. 45). En consonancia con este sentir freireano, tal plan de acción, una vez atendido, pretende producir una doble transformación: la del corazón del profesorado y la de las estructuras verticalizadas que entraban el quehacer universitario y el sistema educativo en general.

\section{Reflexión final}

Leer a Freire y no romperse internamente como profesional "formado" no es posible. Freire, a través de su ideología y su aporte de corte político-intelectual, confronta al ser interno del personal docente y lo invita a realizar un replanteamiento de su propia praxis. Su posición radica en reinventarse continuamente, a través de la revisión del paralelismo y la concordancia entre lo que se piensa, lo que se dice y lo que se hace. Un educador liberado puede convertirse en uno liberador. Este último produce estudiantes activos, reflexivos, críticos, curiosos y, sobre 
todo, ansiosos de provocar un cambio social. Por lo tanto, las acciones transformadoras discutidas en el desarrollo de este ensayo debieran ser incorporadas como herramientas fundamentales a la agenda de clase del profesorado universitario, para provocar una educación liberadora.

En primer lugar, el método dialógico y el empoderamiento serían armas concretas de la pedagogía liberadora, la cual posibilitaría el rompimiento de la cultura del silencio en el colectivo estudiantil. Construir puentes de comunicación entre el cuerpo docente y el estudiantado es tarea propia del quehacer universitario. Acabar con la postura verticalizada del personal docente urge. Por esta razón, las dos acciones transformadoras: el proceso freireano para la realización de lecturas en la clase y las mesas dialógicas facilitarían el diálogo horizontal, provocando en el estudiantado una humanización de sus pensamientos e ideas. En este sentido, Freire (2002) ilustra la importancia del diálogo en la comunicación y da preponderancia a su existir en el acto educativo, de una manera apasionada y clara:

El diálogo es una relación horizontal de A con B. Nace de una matriz crítica y genera criticidad (Jaspers). Se nutre de amor, de humanidad, de esperanza, de fe, de confianza. Por eso, solamente el diálogo comunica. Y cuando los dos polos del diálogo se ligan entre sí, con amor, con esperanza, con fe en el prójimo, se hacen críticos en la procuración de algo y se produce una relación de 'empatía' entre ambos. Solo allí hay comunicación (p. 51).

En segundo lugar, la implementación de una pedagogía de la pregunta es vital en el establecimiento de una educación liberadora. La percepción del estudiantado como un ente que permanece inmutable y vacío apremia ser erradicada. El profesorado debe generar el pensamiento y la desinhibición para cuestionar y analizar. El resultado que se produce al valorar el pensamiento del estudiantado es producto de la horizontalización y la humanización del acto educativo. De lo contrario, la consecuencia sería "una educación sin alegría o inspiración" (Freire y Shor, 2014, p. 250). Y, en definitiva, esto es un efecto que no podemos sentirnos orgullosos de tener en nuestras instituciones de educación superior. La clase o salón invertidos y el método socrático son prácticas transformadoras que iniciarían con erradicar la verticalización del conocimiento. 
En tercer lugar, la reflexión-acción como aspecto vital de este plan de acciones transformadoras desencadenaría el inicio de la pedagogía creativa. Los ateneos didácticos, los cierres efectivos de clase y los espacios de reflexión dentro de una institución educativa son elementos indispensables para estimular una educación emancipadora y responsable, opuesta a la bancaria.

Por último, la alfabetización crítica es la cuarta acción transformadora que se especifica en el plan anterior. En la misma línea argumentativa, la reflexión crítica debe ser el punto de convergencia de los objetivos de un plan de lección universitaria. Es decir, toda actividad de mediación realizada en una clase universitaria debiera estimular el pensamiento crítico, a medida que se realiza. El pensamiento crítico defendido por Freire conlleva no solo la construcción de ideas, sino también la acción. Así como la palabra sin acción es muerta, el pensamiento sin esta sucumbe. De este modo, es a través de los cuestionamientos críticos a lo largo de una lectura y la realización de pequeñas investigaciones derivadas de las consultas hechas en clase que se alfabetiza al estudiantado críticamente.

Por lo demás, debo reiterar que es indispensable abordar esta propuesta del plan de acciones transformadoras de la praxis universitaria con una mirada crítica, pero con una mente abierta. Lamentablemente, debemos admitir que nos hemos equivocado por mucho tiempo. Nos hemos enfocado de manera equivocada y con esto incitado el establecimiento de un aula que no pregunta, una con un estudiantado que no dialoga ni cuestiona. Quizá esta condición del colectivo estudiantil nos ha llenado de confort o tal vez no, pero no hemos tomado las medidas acertadas ni procurado las acciones necesarias que produzcan una reingeniería de nuestras metodologías en el aula. Todo esto nos debería llevar a una reflexión profunda de qué hemos hecho para replantear lo que vamos a forjar; enmendar sin volver a decaer es el único camino que el humano tiene durante toda su vida.

Después de haber analizado parte del legado de Freire, insisto en la urgencia de transformar la praxis universitaria. Es necesario e imperativo iniciar un cambio en los procesos de enseñanza dogmáticos, represivos y verticales, característicos de nuestro sistema educativo, por unos más democráticos, liberadores y horizontales. Que el miedo no sea injustificado y que no paralice (Freire, 1979), sino, más bien, nos impulse a luchar por el ideal de una sociedad más justa, más equitativa 
y democrática; así, producto de este análisis introspectivo, poder decir, al igual que Freire, “...entonces cambi[ó] mi pedagogía, [...] inclinándome por la educación creativa” (Freire y Shor, 2014, p. 42).

\section{Referencias}

Alen, B. (2013). Los ateneos: un dispositivo de acompañamiento a los docentes nóveles. En B. Alen, R. Castellano, R. Hevia Rivas, J. Ramírez, A. Alliaud (eds.), Desarrollo profesional de formadores para el acompañamiento pedagógico de docentes nóveles (pp. 11-24). Montevideo: ANEP, Konrad Adenauer-Stiftung.

Cerletti, A. (2003). La política del maestro ignorante: la lección de Ranciére. Revista Educación y Pedagogía, 15(36), 145-151.

Freire, P. (1979). Educación y mudanza (1. ${ }^{a}$ ed.). Oaxaca de Juárez, Oaxaca: Editorial La mano.

Freire, P. (1982). A importância do ato de ler em três artigos que se completam. Prefacio de Antonio Joaquim Severino. São Paulo, Cortez: Autores Associado.

Freire, P. (1987). Pedagogía do oprimido (17. ${ }^{\text {a }}$ ed.). Rio de Janeiro: Paz e Terra.

Freire, P. (1992). Pedagogía del oprimido. Madrid: Siglo XXI Editores.

Freire, P. (1993). Professora sim, tia nao. Sao Paulo: Olho d'Agua.

Freire, P. (1996). Pedagogía de la autonomía. Saberes necesarios de la práctica educativa. Sao Paulo: Paz y Tierra.

Freire, P. (2002). Cartas a quienes pretenden enseñar. México: Siglo XXI Editores.

Freire, P. (2013). Pedagogía de la pregunta. Buenos Aires: Siglo XXI Editores.

Freire, P. y Shor, I. (2014). Miedo y osadía. La cotidianidad del docente que se arriesga a practicar una pedagogía transformadora. Buenos Aires: Siglo XXI Editores.

Gadamer, H. (1994). Verdad y método. Salamanca: Editorial Sígueme.

Gramsci, A. (1972). Introducción a la filosofia de la praxis. Barcelona: Península.

Hegel, G. W. F. (1991). Escritos pedagógicos. Madrid: FCE.

Kant, I. (1983). Pedagogía. Madrid: Akal.

Masi, A. (2008). El concepto de praxis en Paulo Freire. En P. Freire (ed.), Contribuciones para la pedagogía (pp. 75-82). Buenos Aires: CLACSO. 
Mattis, K. (2014). Flipped classroom versus traditional textbook instruction: Assessing accuracy and mental effort at different levels of mathematical complexity. Technology, Knowledge and Learning, 24(67), 231-248. Doi: https://doi.org/10.1007/ s10758-014-9238-0

Sáiz, A. (2003). Freire: comunicación y filosofia. México: Ediciones Acatlán.

Santos, M. (2008). Ideas filosóficas que fundamentan la pedagogía de Paulo Freire. Revista Iberoamericana de Educación, 46, 155-173.

Streck, D.; Redín, E. y Zitkoski, J. (2010). Diccionario Paulo Freire (2. ${ }^{a}$ ed., rev., amp., 1. ${ }^{\mathrm{a}}$ reimp.). Brasil, Belo Horizonte: Auténtica Editora.

UniversidadNacional.(2012).Modelopedagógico. Recuperado dehttp:// www.documentos.una.ac.cr/bitstream/handle/unadocs/1763/modelo_pedagogico_UNA.141. pdf? sequence $=3$ \&isAllowed $=y$

Zetina-Esquivel, E. y Piñón-Rodríguez, P. (2017). El método socrático en los programas educativos actuales: una propuesta de Martha C. Nussbaum. La Colmena, 91, 79-90. Recuperado de https:// lacolmena.uaemex.mx/article/view/5202 
